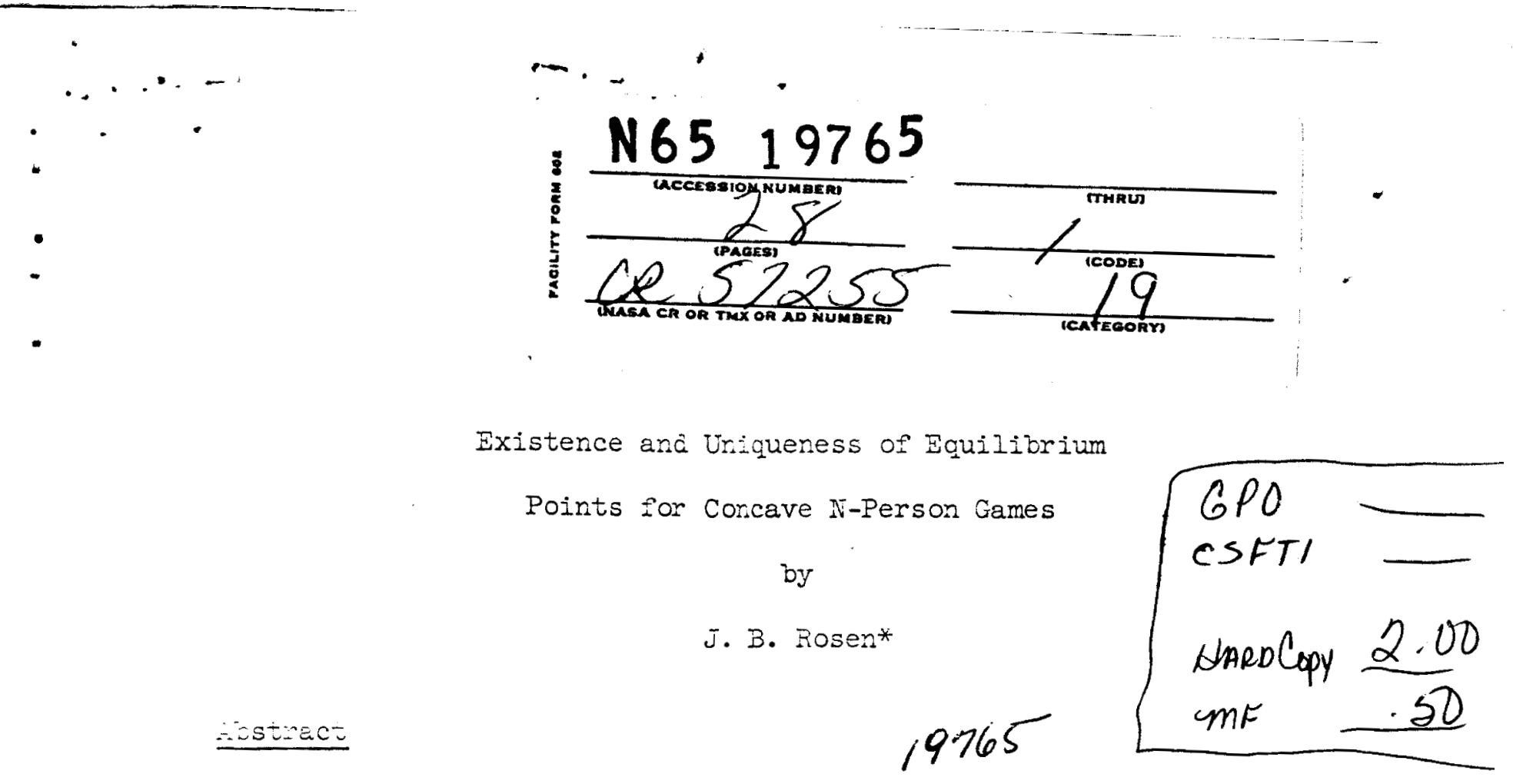

A constrained $n$-person game is consiaered in which the constraints For each player, as well as his payoff function, may depend on the suraitiy of every player. The existence of an equilibrium point for Fich a game is shown. By requiring appropriate concavity in the payof uncions a concave game is defined. It is proved that there is a unique equilibrium point for every strictly concave game. A dynamic model for ronecuilibrium situations is proposea. This model consists of a system of aifferential equations wich specty the rate of change of each Elayer's strategy. It is sncws that for a strictly concave game the system is globally asymptotically stable with respect to the unique a equilibrium point of the game. Finally, it is shown how a gradient. methoa suitable for a concave mathematical programming problem can be üsea to finc the equilibrium point for a concave game.<smiles>CCCCCCCCCCCCCCCCCCCC</smiles>

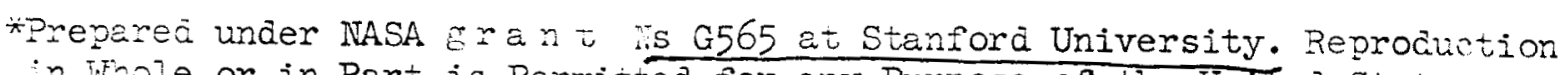
in Whole or in Part is Permitted for any Purpose of the United States Government. 
2. Zntrainction.

The concept of an equilibrium point for an n-person game was incrociced by Fasi $[20,1]]$ and the existence of such points proved under certain assumptions on each piayer's strategy space and corresponding payofí function. He showed that if each player is restricted to a simplex in his own strategy space ano if the payof functions are bilinear functions of the strategies, then an eciuizibrium poinc exists. Tris result has been generalized to an abstract economy by Arrow anā Jejreu [I] and McKenzie [9], where each player's strategy space may àepená on the stritegy of the other players.

Tris more general problem is considered here. Specilically, it is only recuired that every joint strategy, represented by a point in the product space of ite ināividual strategy spaces, lie in a convex, closed and boundeã region R in the proöuct space and that each player's payoff function $\varphi_{i}, i=1, \ldots n$, we concave in his own strategy. The existence of an equilibrium point for this concave $n$-person game is shown in Theorem 1 , using a mapping of $R$ into $R$ anc̄ tre Kaikutani jixeà point theorem [4].

One of the difficulties which has limited the usefulness of the concept of ar equiliorium point for an n-person game is the lack of uniqueness of such points, as shown by the fact that mary games possess an infinite number of ecuilibrilim points (for example, see shapley [12]). This difficulty is overcome by requiring that the payoff functions satisfy' an additional concavity recuirement wish is callea diagonal strict concavity. With this adaitional recuirement it is shown in Theorems 2, 3 and 4 that every concave n-person E.... has a unique equilibrium point. Theorem 2 shows uniqueness for a game Win orthozonal constraint sets, that is, where $R$ is the direct product of the inaiviaul player's strategy spaces. In Theorems 3 the more general case of 


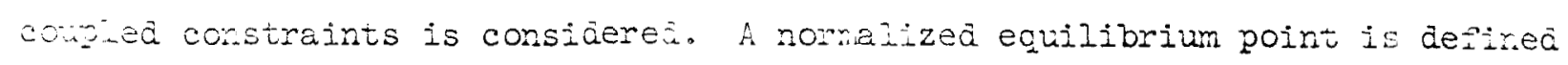
In terms of a specifiea positive constant $r_{i}$ for each player, witici determines

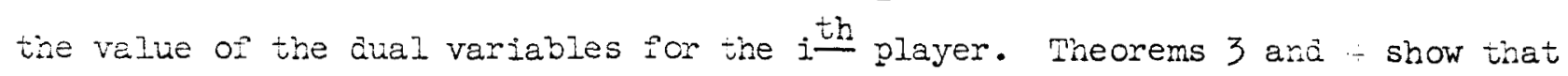
a unique normalizea equilibrium poing exists for each specified value of the perameters $r_{i}$. The monotone behavior at the equilibrium point of the payoff ancition $\varphi_{i}$ with respect to $r_{i}$ is shown in Theorem 5 . Section 3 is completed by giving a sufficient condition for diagonal strict concavity in temms of certain Hessian matrices oi the ${ }_{i}$. The interesting case where each Is izinear in the strategies is discussea to illustrate this condition. The bimatrix game $[7,8]$ is a special case of tris bilinear payo i function. In Section 4 we consicer a reasonable aynamic model of the $x$-person concave game. It is assumed that if the game is not at equilibrium each player will atuemit to change his orm strategr so as to obtain the maximum rate of change 0 ins own payor function with respect to a change in his own strategy. It is shom that the system of differential equations obtained in this way has the property that every solution starting in $R$ remains in $R$ (Theorem 7 ). The subility of the system is considered in Theorems 8 and 9 . It is shown that Sen concavity conaitions sufficient for uniqueness are satisfied the system of aifferential equations is globaliy asymptotically stable. Furthermore, starting at any feasible point in the strategy space $R$, the system of aifierential equations will always converge to the unique equilibrium point of ine original $n$-person concave game. Thus the dynamic model and the concave Eare have the same unique equilibrium point. The stability proof uses the unere of the norm of the right hand side of the differential equations as a Ziapunov function to show that the norm approaches zero. The stability of a àjzerent dynamic model of a competitive equilibrium representea by a system of äjaerential equations has previously been investigated [13, 19]. 
In seciion 5 it is show that the unique equilibrium point to tre concave jame can be founa computationally by using a gracient method suitable for a concave mathematical programming problem [17, 18]. This may be considered as a Eeneralization of the well known relationship between the two-person zerosum game and linear programming [15]. It should also be noted that the general concave constrainea maximization problem is obtained for the case $\mathrm{n}=1$, so that such a problem may be considered as a special case of the $n$-person concave game. For this special case of $n=1$, the results of Sections 2 and 3 reduce to knom resuits. However, the resuits of Section 4, in particular Theorem 7 , appear to be new even for $\mathrm{n}=1$. 


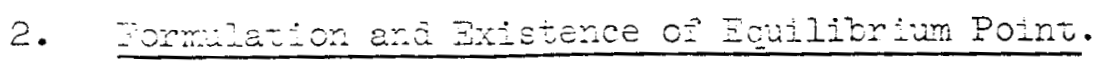

The concave n-perso ame to be consiciered is aescribed in terms or the ir.aiviulial stratea vector for each of the $n$ players. The strategy of the ith player is represented by the vector $x_{i}$ in the Eucliaian space $\Sigma^{m_{i}}, i=1, \ldots$. The vector $x \in E^{m}$ then denoter he simidianeous strategies of dil players, where $\mathrm{E}^{\mathrm{mi}}$ is the product space $\bar{L}^{2} \times \mathrm{E}^{\mathrm{m}_{2}} \ldots \times \mathrm{E}^{\mathrm{m}_{\mathrm{n}}}$ and $\mathrm{m}=\sum_{i=1}^{r_{1}} m_{2}$. The alLowes stratefies will be limitea by the requirement that $x$ be seircteo from a cunvex, closea ana boincea set $R C \mathrm{I}^{\mathrm{m}}$. If we denote by $P_{i}$ the projection of $R$ or $I^{m}$, we will also consiäer the contex, closed and bounded product set $S \supseteq E$, ziver by $S=P_{I} \times P_{2} \ldots \times P_{n}$. This is illustrated in Fis。 $I$ ior $n=2$.

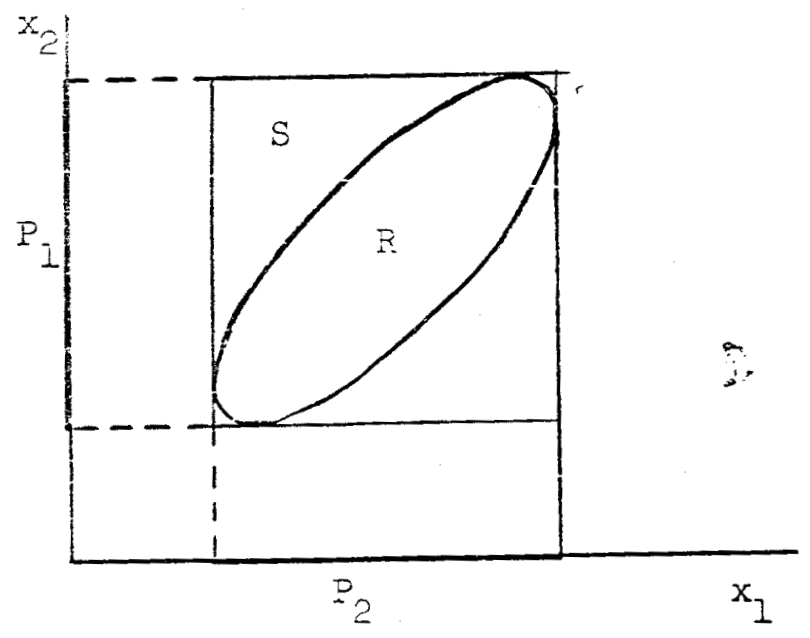

Figure I

In most game theory papers consiaeration is limited to the case where each clayer's strategy $x_{i}$ is restrictea to a convex set $k_{i} \subset E_{i}$ in his own strategy space. For example, Ir Nash $[0,11]$ the set $R_{i}$ is the simplex in $T^{2}-$ In this special case where the contraint sets are orthogoral we have $P_{1}=R_{2}$, so that $R=S=R_{2} \times F_{2} \ldots \times R_{n}$. In the general case where $R \subset S$ we wili say that $R$ is a couried constraint set. 
Tre payozif function for the th player aepenas on the strategies of ali the over piayers as well as his own strategy, ano is given by the furction $\varphi_{i}(x)=\varphi_{i}\left(x_{1}, \ldots x_{i}, \ldots x_{n}\right)$. It will be assumea that for $x \in S, \varphi_{i}(x)$ is continuous in $x$ and is concave in $x_{i}$ for each fixed value of $\left(x_{2}, \ldots x_{i-I}, x_{i+1}, \ldots x_{n}\right)$. With this formulation an equilibrium point of the r-person concave game is given by a point $x^{\circ} \in R$ such that

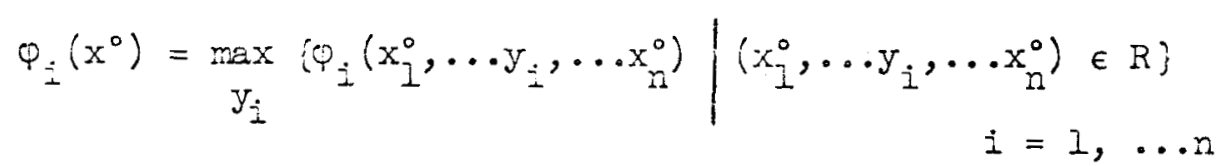

At such a point no player can increase his payoff by a unilateral change in his strategy.

The results to follow make use of the function $p(x, y)$ defined for $(x, y) \in \bar{K} \times \bar{x} \quad$ Dy

$$
p(x, y) \equiv \sum_{i=1}^{n} \rho_{i}\left(x_{1}, \ldots y_{i}, \ldots x_{n}\right)
$$

Wo oberve that for $(x, y) \in R \times R$ we have $\left(x_{1}, \ldots y_{i}, \ldots x_{n}\right) \in S, i=1, \ldots n$, sc that $p(x, y)$ is continuous in $x$ anà $y$ and is concave in $y$ for every fixes $x$, for $(x, y) \in R \times R$. We now prove the existence theorem for the concave $n$-person game.

The orem I

An equilibrium point exists for every concave n-person game. Prooi:

Consiàer the point to set mapping $x \in R \rightarrow \Gamma x \subset R$, given by

$$
\Gamma x=\left\{y \mid \rho(x, y)=\max _{z \in R} \rho(x, z)\right\}
$$


It fojiows ixom the continuity of $\rho(x, z)$ and the concavity in $z$ of $p(x, z)$ For lixec $x$, that $\Gamma$ is an upper semicontinuous mapping wich rapi each point of the convex, compact set $R$ into a closed convex subset of $R$. Ther by the Kakutani fixed point theorem $[4,5]$ there exists a point $x^{\circ} \in R$ such that $x^{\circ} \in \Gamma x^{\circ}$, or

$$
p\left(x^{0}, x^{0}\right)=\max _{z \in R} p\left(x^{0}, z\right)
$$

The fixea point $x^{\circ}$ is an equilibrium point satisfying (2.1). For suppose that It were not. Then, say for $i=h$, there would be a poirt $x_{l}=\vec{x}_{2}$ such tiat $\bar{x}=\left(x_{2}^{0}, \ldots \bar{x}_{2}, \ldots x_{n}^{0}\right) \in R$ and $\varphi_{2}(\bar{x})>\varphi_{2}\left(x^{0}\right)$. But then we have $p\left(x^{\circ}, \bar{x}\right)>p\left(x^{\circ}, x^{\circ}\right)$ which contracicts $(2.4)$ 


\section{Uniqueness of Equiliorium Point.}

In oncer to discuss the uniqueness of an equilibrium point we must cescrive the convex set $R$ more explicitiy. For the general couplea constraint set where $R C S$, we will describe $R$ by means of the mapping $h(x)$ of $\mathbb{E}^{\mathrm{m}} \rightarrow E^{\mathrm{k}}$, where each component $h_{j}(x), j=1, \ldots k$ of $h(x)$ is a concave function of $x$. It is assumed that

$$
R=\{x \mid h(x) \geq 0\}
$$

is norvoia and bouncea. It follows from the concavity of the $h_{j}\left(x_{j}\right)$ that the ciosea set $R$ is convex. For the orthogonal constraint set $R=S=R_{1} \times R_{2} \ldots \times R_{n}$, we consicer the nonvoid and boundea sets

$$
R_{i}=\left\{x_{i} \mid h_{i}\left(x_{i}\right) \geq 0\right\}, i=1, \ldots n
$$

wisere each component $h_{i j}\left(x_{i}\right), j=1, \ldots k_{i}$, is a concave function of $x_{i}$,

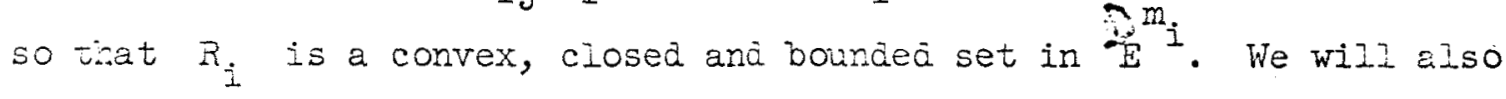
assume that the set $R$ contains a point which is strictly interior to every nonlinear constraint, that is, $\exists \bar{x} \in R$, such that $h_{j}(\bar{x})>0$ for every nonitinear constraint $h_{j}(x)$. This is a sufficient condition ior the satisfaction of the Kunn-Thucker constraint qualification [ 2 ].

We wish to use the aifferentiai form of the necessary and sufficient Kunn-Tucker conatitions for a constrainea maximum [ 6 ]. We therefore make the adaitional assumption that the $h_{j}(x)$ possess continuous first derivatives for $x \in R$. We also assume that for $x \in R$ the payoff function $O_{i}(x)$ For the $i$ th player possesses continuous first derivatives with respect to the components of $x_{i}$. For any scalar function $\varphi(x)$ we denote oy $\nabla_{i} \varphi(x)$ the gradient. with respect to $x_{1}$ of $\varphi(x)$. Thus $\nabla_{i} \varphi(x) \in E^{m_{1}}$. 
The Kun-Tucker conditions equivalent to (2.1) with $R$ given oy (3.1) can now be stated as Iollows:

$$
h\left(x^{0}\right) \geq 0
$$

and for $i=I, \ldots n, \exists u_{i}^{0} \geq 0, u_{i}^{0} \in E^{k}$, such that

$$
u_{i}^{\circ} h\left(x^{0}\right)=0
$$

$\operatorname{ard}$

$$
\varphi_{i}\left(x^{0}\right) \geq \varphi_{i}\left(x_{1}^{0}, \ldots y_{i}, \ldots x_{n}^{0}\right)+u_{i}^{0}{ }^{\prime}\left(x_{1}^{0}, \ldots y_{i}, \ldots x_{n}^{0}\right)
$$

Since $\varphi_{i}(x)$ and $h_{j}(x)$ are concave and differentiabie, the inequality $(3.5)$ is equivalent to

$$
\nabla_{i} \varphi_{i}\left(x^{0}\right)+\sum_{j=I}^{k} u_{i j}^{0} \nabla_{i} n_{j}\left(x^{0}\right)=0, \quad i=1, \ldots n
$$

We will also use the following relation winich holds as a result of the concavity of $h_{j}(x)$. For every $x^{0}, x^{\prime} \in R$ we have

$$
h_{j}\left(x^{1}\right)-h_{j}\left(x^{0}\right)_{2} \leq\left(x^{0}-x^{0}\right) \nabla h_{j}\left(x^{0}\right)=\sum_{i=1}^{n}\left(x_{i}^{1}-x_{i}^{0}\right)^{\prime} \nabla_{i} h_{j}\left(x^{0}\right)
$$

A weighted nonnegative sum of the functions $\varphi_{i}(x)$ is given by

$$
\sigma(x, r)=\sum_{i=1}^{n} r_{i} \varphi_{i}(x), \quad r_{i} \geq 0
$$

For each nornegative vector $r \in E^{n}$. For each fixed $r$, a relatea mapping $\delta(x, r)$ of $\mathrm{E}^{\mathrm{m}}$ into itself is defined in terms of the gradients $\nabla_{i} \varphi_{i}(x)$ by 


$$
g(x, r)=\left(\begin{array}{c}
r_{2} \nabla_{1}(x) \\
r_{2} \nabla_{2} \varphi_{2}(x) \\
r_{n} \nabla_{n} \varphi_{n}(x)
\end{array}\right)
$$

Ar important property of $\sigma(x, x)$ is given by the following Definition

The function $\sigma(x, r)$ will be called diagonally strictly concave for $x \in R$ and fixed $x \geq 0$, if for every $x^{\circ}, x^{\prime} \in R$ we have

$$
\left(x^{\prime}-x^{0}\right)^{\prime} g\left(x^{0}, r\right)+\left(x^{0}-x^{0}\right)^{\prime} g\left(x^{1}, r\right)>0
$$

As shown later, a sufficient condition that $\sigma(x, r)$ be diagonally suictiy concave is that the symetric matrix $\left[G(x, r)+G^{-}(x, r)\right]$ is negative aefinite for $x \in R$, where $G(x, r)$ is the Jacobian with respect to $x$ of $g(x, r)$.

We first give the uniqueness theorem for orthogonal constraint sets where $R=S$.

Tiseorem 2

Ii $\sigma(x, r)$ is diagonally strictly concave for some $r=\bar{r}>0$, then the equilibrium point $x^{\circ}$ satisfying (2.I) is unique.

Proof:

Assume there are two aistinct equilibrium points $x^{\circ}$ and $x^{\prime} \in R$, each $0:$ which satisfies (2.1). Then by the necessity of the Kuhn-Tucker conaitions we have for $l=0$, $I$ anc $i=1, \ldots$,

$$
\begin{gathered}
h_{i}\left(x_{i}^{l}\right) \geq 0 \\
\exists u_{i}^{l} \geq 0, u_{i}^{l} \in E^{k}, \text { such that } \\
-10-
\end{gathered}
$$




$$
\begin{gathered}
u_{i}^{\ell^{\prime}} h_{i}\left(x_{i}^{l}\right)=0 \\
\nabla_{i} p_{i}\left(x^{2}\right)+\sum_{j=1}^{k_{i}} u_{i j}^{l} \nabla_{i} h_{i j}\left(x_{i}^{2}\right)=0
\end{gathered}
$$

We muitiply $(3.13)$ by $\bar{r}_{i}\left(x_{i}^{\prime}-x_{i}^{0}\right)^{\prime}$ for $l=0$ and by $\bar{r}_{i}\left(x_{i}^{0}-x_{i}^{0}\right)^{\prime}$ for $l=1$, anà sum on i. This gives

$$
\beta+\gamma=0
$$

where

$$
\beta=\left(x^{1}-x^{0}\right)^{\prime} g\left(x^{0}, \bar{r}\right)+\left(x^{0}-x^{\prime}\right)^{0} g\left(x^{\prime}, \bar{r}\right)
$$

anç

$$
\begin{aligned}
\gamma & =\sum_{i=1}^{n} \sum_{j=1}^{k_{i}} \bar{r}_{i}\left\{u_{i j}^{0}\left(x_{i}^{\prime}-x_{i}^{0}\right)^{\prime} \nabla_{i} h_{i j}\left(x_{i}^{0}\right)+u_{i j}^{\prime}\left(x_{i}^{0}-x_{i}^{\prime}\right) \nabla_{i} h_{i j}\left(x_{i}^{\prime}\right)\right\} \\
& \geq \sum_{i=1}^{n} \sum_{j=1}^{k_{i}} \bar{r}_{i}\left\{u_{i j}^{0}\left[h_{i j}\left(x_{i}^{0}\right)-h_{i j}\left(x_{i}^{0}\right)\right\}+u_{i j}^{0}\left[h_{i j}\left(x_{i}^{0}\right)-h_{i j}\left(x_{i}^{0}\right)\right]\right\} \\
& =\sum_{i=1}^{n} \bar{r}_{i}\left\{u_{i}^{0} h_{i}\left(x_{i}^{0}\right)+u_{i}^{0} h_{i}\left(x_{i}^{0}\right)\right\}
\end{aligned}
$$

We inequality follows from the concavity of the $h_{i j}(x)$ and $(3.7)$, and the last relation from (3.12). Then from (3.11) we have that $\gamma \geq 0$. Since $\sigma(x, \bar{r})$ is diagonally strictly concave it follows from $(3.10)$ that $\beta>0$. But this contradicts (3.14), so that we cannot have two distinct equilibrium poinus ana therefore $x^{0}$ is unique.

We now consider the general case where $R$ is a couplea constraint set and is Eiver by (3.i). The values of the nonnegative multipliers $u_{i}^{0}, i=1, \ldots n$ given by the Kubn-Thucker conditions at an equilibrium point will, in Eeneral, 
not be relatea to each other. We will consiuer a special kinà of ecuilibrium point such that each $u_{i}^{0}$ is given gr

$$
u_{i}^{0}=u^{0} / r_{i}, \quad i=1, \ldots . n
$$

for some $r>0$, and $u^{\circ} \geq 0$. We will call this a normalized equilibrium 20ins.

reonem 3

There exists a normalizea equilibrium point to a concave n-person game for Erery specizied r $>0$.

Proof:

For a iixed value $r=\bar{r}>0$, let

$$
\rho(x, y, \bar{r})=\sum_{i=1}^{n} \bar{r}_{i} \varphi_{i}\left(x_{1}, \ldots y_{i} \ldots x_{n}\right)
$$

Using the fixed point theorem as in Theorem 1 , therepexists a point $x^{\circ}$ such tinat

$$
\rho\left(x^{\circ}, x^{\circ}, \bar{r}\right)=\max _{y}\left\{\rho\left(x^{\circ}, y, \bar{r}\right) \mid h(y) \geq 0\right\}
$$

Then by the necessity of the Kunn-Tucker conaitions, $h\left(x^{\circ}\right) \geq 0$, and $\exists u^{\circ} \geq 0$, such that ${ }^{\circ} u^{\circ} h\left(x^{\circ}\right)=0$ and

$$
\bar{r}_{i} \nabla_{i} \varphi_{i}\left(x^{0}\right)+\sum_{j=1}^{k} u_{j}^{0} \nabla_{i} n_{j}\left(x^{0}\right)=0, \quad i=1, \ldots n
$$

Bui these are just the conaition $(3.3),(3.4)$ and $(3.6)$, with $u_{i j}^{0}=u_{j}^{0} / \bar{r}_{i}$, or $u_{i}^{0}=u^{\circ} / \bar{r}_{i}$, which are sup $u^{\prime}$ ient to insure that $x^{0}$ satisfies (2.I). $x^{\circ}$ is tinerefore a normalized equilibrium point for the specifiec value of $r=\bar{r}$. 


\section{meorem 4}

Let $\sigma(x, r)$ De diagonaliy strictly concave for every $r \in Q$, where $Q$ Is a convex subset of the positive orthant of $\mathrm{E}^{\mathrm{n}}$. Then for each $r \in \mathrm{Q}$ there is a unique wormalized equilibrium point.

Proos:

Assume that for some $r=\bar{r} \in Q$ we have two normalized equilibrium points $x^{0}$ and $x^{\prime}$. Then we have for $l=0,1$ and $i=1, \ldots n$,

$$
\begin{gathered}
k\left(x^{l}\right) \geq 0 \\
\exists u^{l} \geq 0, u^{l} \in \equiv^{k}, \text { such that } \\
u^{\ell} h\left(x^{l}\right)=0 \\
\bar{r}_{i} \nabla_{i} \varphi_{i}\left(x^{l}\right)+\sum_{j=1}^{k} u_{j}^{l} \nabla_{i} h\left(x^{l}\right)=0
\end{gathered}
$$

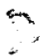

We miltipiy $(3.23)$ by $\left(x_{i}^{0}-x_{i}^{0}\right)^{\prime}$ for $l=0$ and by $\left(x_{i}^{0}-x_{i}^{1}\right)$ for $l=1$, and sim on $i$. As in the proof of Theorem 2 this gives $\beta+\gamma=0$, where $\beta$ is given by $(3.15)$ and

$$
\begin{aligned}
\gamma & =\sum_{j=1}^{k} \sum_{i=1}^{n}\left\{u_{j}^{0}\left(x_{i}^{\prime}-x_{i}^{0}\right)^{\prime} \nabla_{i} h\left(x^{0}\right)+u_{j}^{\prime}\left(x_{i}^{0}-x_{i}^{\prime}\right)^{\prime} \nabla_{i} h\left(x^{\prime}\right)\right\} \\
& \geq u^{\circ}\left[h\left(x^{\prime}\right)-h\left(x^{0}\right)\right]+u^{\prime}\left[h\left(x^{0}\right)-h\left(x^{\prime}\right)\right] \\
& =u^{\circ} h\left(x^{\prime}\right)+u^{\prime} h\left(x^{0}\right) \geq 0
\end{aligned}
$$

Taen since $\sigma(x, \bar{r})$ is diagonally strictly concave we have $B>0$, which contradicts $\beta+\gamma=0$ and proves the tifeorem. 
We will now investigate the dependence of the normalizea equilibrium point on the vaiue of $r$ for the generai case where $R$ is a couplea constraint set. For an orthogonal constraint set it follows from Theorem 2 that if $\sigma(x, r)$ is aiagonally strictly concave for some $r=\bar{r}>0$, the equilibrium point $x^{0}$ is triependent of $r$. On the other hand it is not difficult to construct a simple. example with a coupled constraint set (see Fig. 2) where the equilibrium point $\mathrm{x}^{\circ}$ àoes àepenà on $\mathrm{r}$.

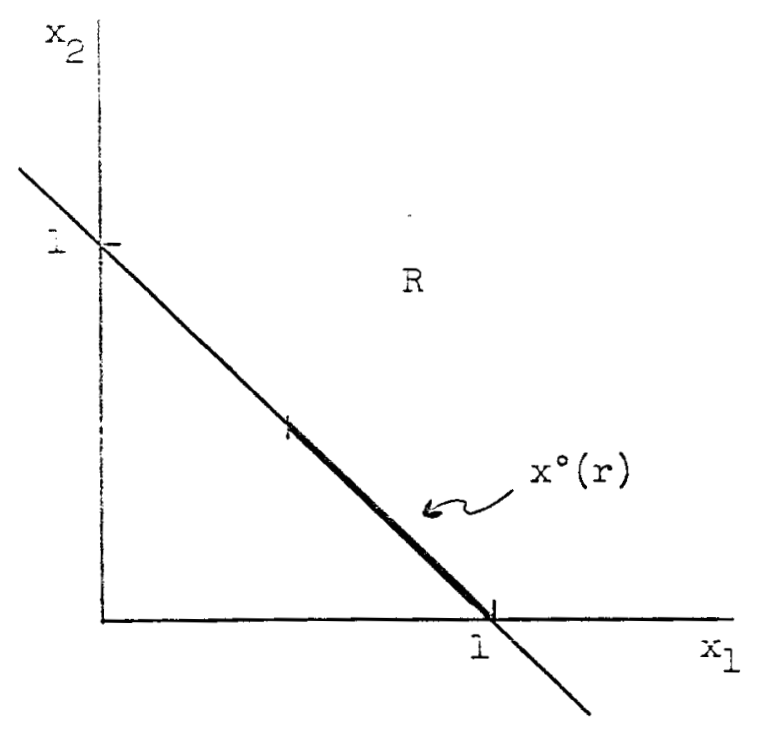

$$
\begin{aligned}
& \varphi_{1}(x)=-\frac{1}{2} x_{1}^{2}+x_{1} x_{2} \\
& \varphi_{2}(x)=-x_{2}^{2}-x_{1} x_{2} \\
& n_{1}(x)=x_{1} \geq 0 \\
& h_{2}(x)=x_{2} \geq 0 \\
& h_{3}(x)=x_{1}+x_{2}-1 \geq 0 \\
& \varphi_{1}\left(x^{0}\right)=\max _{x_{1}}\left\{\varphi_{1}\left(x_{1}, x_{2}^{0}\right) \mid h\left(x_{1}, x_{2}^{0}\right) \geq 0\right\}=x_{1}^{0}(1 . \\
& \varphi_{2}\left(x^{0}\right)=\max _{x_{2}}\left\{\frac{\varphi_{2}}{2}\left(x_{1}^{0}, x_{2}\right) \mid h\left(x_{1}^{0}, x_{2}^{0}\right) \geq 0\right\}=x_{1}^{0}- \\
& x_{1}^{\circ}=\left\{\begin{array}{cc}
1, & r_{1} \leq r_{2} \\
\frac{r_{1}+2 r_{2}}{2 r_{1}+r_{2}}, & r_{2}>r_{2}
\end{array}\right\}, \quad x_{2}^{0}=1-x_{1}^{0}
\end{aligned}
$$

In such a case we will now show that in a certain sense the equilibrium value of $\varphi_{i}$ is a monotone increasing function of $r_{i}$.

Theorem 5

Let $\sigma(x, r)$ be diagonaliy strictiy concave for $r \in Q$. Let $r^{\circ}, r^{\prime} \in Q$ be such that $r_{i}^{0}=r_{i}^{0}, \quad i \neq q$ and $r_{q}^{1}>r_{q}^{0}$ Let $x^{0}$ and $x^{\prime}$, with $x^{\prime} \neq x^{\circ}$, be the corresponding unique nomalized equilibrium points. Then the airectional derivative of $\varphi_{q}\left(x^{0}\right)$ along the ray $\left(x_{q}^{\prime}-x_{q}^{0}\right)$ is positive. 
Proos:

Let $u^{\circ}$ and $\therefore$ be the ... pilers corresponding to the nomiaitzed equilibrium points $x^{\circ}$ and $x$. Then for $l=1$. ar $i=1, \ldots \ldots$, ana for $2=0$ an $\bar{a} \neq q$, the relation (3.21), (3.22) and (3.23) are satisriad witis $\bar{r}_{i}=r_{i}$. For $\ell=0$ and $i=q$, we have

$$
\left(r_{q}^{0}-r_{q}^{i}\right) \nabla_{q} q_{q}\left(x^{0}\right)+r_{q}^{i} \nabla_{q} q_{q}\left(x^{0}\right)+\sum_{j=1}^{k} u_{j}^{0} \nabla_{q} h\left(x^{0}\right)=0
$$

Nivitiplying by $\left(x_{i}^{1}-x_{i}^{0}\right)^{\prime}$ for $l=0$ ana $\left(x_{i}^{0}-x_{i}^{\prime}\right)^{\prime}$ for $l=1$, anà summing now gives

$$
\left(r_{q}^{0}-r_{q}^{1}\right)\left(x_{q}^{i}-x_{q}^{0}\right)^{\prime} \nabla_{q} q_{q}\left(x^{0}\right)=-(\beta+\gamma)<0
$$

or since $r_{q}^{\prime}>r_{q^{\prime}}^{\circ}$

$$
\left(x_{q}^{1}-x_{q}^{0}\right)^{\prime} \nabla_{q}^{Q} q\left(x^{0}\right)>0
$$

But tris is just the airectionai aerivative of $\varphi_{\mathrm{q}}\left(\mathrm{x}^{\circ}\right)$ along the $r=y$ $\left(x_{q}^{i}-x_{q}^{0}\right)$

A useful interpretation of Theorem 5 is obtained by observing that if ${ }_{{ }_{\mathrm{C}}}(x)$ has bounded $2 \frac{\text { nd }}{2}$ partial derivatives and $\left\|x_{q}^{\prime}-x_{q}^{0}\right\|$ is sufficiently small then it 30 llows from (3.27) that $\oint_{q}(\bar{x})>\varphi_{q}\left(x^{0}\right)$, where $\bar{x}=\left(x_{1}^{0}, \ldots x_{q}^{1}, \ldots x_{n}^{0}\right)$. Since $x^{\circ}$ is an equilibrium point $\bar{x}$ cannot be a feasible point, and the vallie of $\varphi_{\mathrm{q}}(\mathrm{x})$ may decrease as $\mathrm{x}$ goes from the infeasible point $\bar{x}$ to the new (feasible) equilibrium point $x^{2}$, as illustrated in Fig. 3 . Because of the diazonal concavity property of $\varphi_{i}(x)$, the depenaence of $\varphi_{q}(x)$ on $x_{c}$ will usually aominate its b, mience on $x_{i}, i \neq q$. Therefore, it will usuilly be true that $\varphi_{q}\left(x^{\prime}\right)>\varphi_{q}\left(x^{0}\right)$. This is illustrated by the example of IIE. 2, where it is easy to show that both $\frac{\partial \phi_{1}}{\partial r_{1}}$ and $\frac{\partial \varphi_{2}}{\partial r_{2}}$ are nonnegative. 


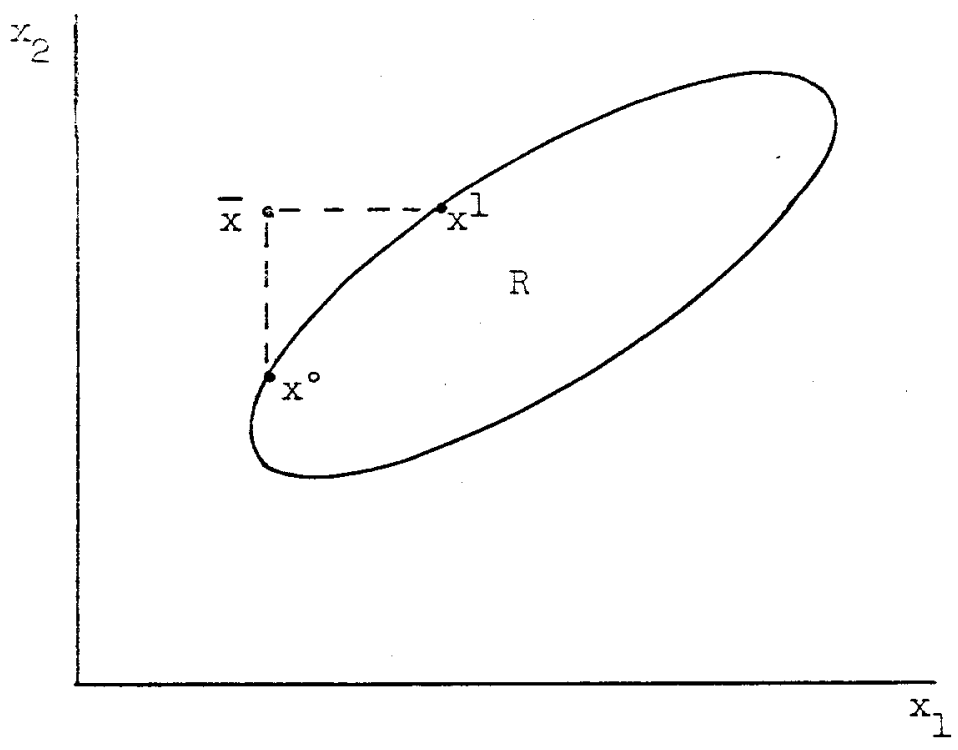

Figure 3

We complete this section by giving a sufficient conaition on the functions $Q_{i}(x)$, which insures that $\sigma(x, r)$ is diagonally strictly concave. The conition is given in terms of the mxm matrix $G(x, r)$ which is the Jacobian $O g(x, r)$ for fixed $r>0$. That is, the $j^{\text {th }}$ column of $G(x, r)$ is $\frac{\partial g(x, r)}{\partial x_{j}}, \quad j=1, \ldots m$, where $g(x, n)$ is aefineç by (3.9).

rasem 6

A surficient condition that $\sigma(x, r)$ be aiagonally strictly concave for $x \in R$ and fixed $r=\vec{r}>0$, is that the symmetric matrix $\left[G(x, \vec{r})+G^{\prime}(x, \vec{r})\right]$ be negative aefinite for ${ }^{*} \mathrm{x} \in \mathrm{R}$.

Proc: :

Let $x^{\circ}, x^{\prime}$ be any two aistinct points in $R$, and let $x(\theta)=\theta x^{\prime}+(1-\theta j x$ so that $x(\theta) \in R$ for $0 \leq \theta \leq 1$. Now since $G(x, \bar{r})$ is the Jacobian of $g(x, \bar{r})$ we have

$$
\begin{aligned}
\frac{\hat{\mathrm{ag}}(\mathrm{x}(\theta), \bar{r})}{\mathrm{d} \theta}=G(x(\theta), \bar{r}) & \frac{\overline{\mathrm{ax}}(\theta)}{\mathrm{a}}=G(x(\theta), \bar{r})\left(x^{3}-x^{0}\right) \\
& -16-
\end{aligned}
$$


0

$$
g\left(x^{\prime}, \bar{r}\right)-g\left(x^{0}, \bar{r}\right)=\int_{0}^{1} G(x(\theta), \bar{r})\left(x^{\prime}-x^{0}\right) d \theta
$$

Nuitiplying both siàes by $\left(x^{0}-x^{\prime}\right)^{\prime}$ gives

$$
\begin{gathered}
\left(x^{0}-x^{1}\right)^{\prime} g\left(x^{1}, \bar{r}\right)+\left(x^{1}-x^{0}\right)^{\prime} g\left(x^{0}, \bar{r}\right)=-\int_{0}^{1}\left(x^{\prime}-x^{0}\right)^{\prime} G(x(\theta), \bar{r})\left(x^{1}-x^{0}\right) \text { a } \theta \\
=-\frac{1}{2} \int_{0}^{1}\left(x^{1}-x^{0}\right)^{\prime}\left[G(x(\theta), \bar{r})+G^{\prime}(x(\theta), \bar{r})\right]\left(x^{\prime}-x^{0}\right) \text { d } \theta>0(3.30)
\end{gathered}
$$

Wich shows that $(3.10)$ is satispiea.

Tre interesting case where $\varphi_{i}(x)$ is bilinear in the strategies $x_{j}$ emphasizes an important relation detween this condition and a stability matrix. We Iet

$$
\varphi_{i}(x)=\sum_{j=1}^{n}\left[e_{i j}^{\prime}+x_{i}^{\prime} C_{i j}\right] x_{j}, \quad i=1, \ldots n
$$

Where $e_{i j}$ is a constant vector in $E^{m_{j}}$ and $C_{i j}$ is an $m_{i} \times m_{j}$ conzint mavizix. The bimatrix game $[7,8]$ is a special case of (3.31) with $\therefore=a, e_{i j}=0, c_{11}=c_{22}=0$ and $c_{12} \neq 0, c_{21} \neq 0$. The two-person zero-sum same is a Iurther specialization with $\mathrm{C}_{21}=-\mathrm{C}_{12}^{\prime}$.

From definition (3.9) $\mathrm{O} \overrightarrow{\mathrm{g}} \mathrm{g}(\mathrm{x}, \mathrm{r})$ and $\mathrm{G}(\mathrm{x}, \mathrm{r})$ as its Jácobian matrix, we ototain

$$
G(x, r)=D C
$$

where $C$ is the $m \times m$ constant metrix

$$
C=\left(\begin{array}{cccc}
2 c_{11} & c_{12} & \cdots & c_{1 n} \\
c_{21} & 2 c_{22} & & \\
\dot{0} & & & \\
c_{n 1} & & & \\
c_{n n} & & & 2 c_{n}
\end{array}\right)
$$


ance $D$ is the diagonal positive definite matrix $D=$ Giag $\left\{r_{i}\right\}$. For this biinean case it follows from wheorems 2 and 6 that we have uniqueness if there exists some $\bar{r}>0$ such that

$$
\bar{D} C+C^{\prime} \bar{D}=-I
$$

where $\bar{D}=\overline{a i a g}_{0}\left\{\bar{r}_{i}\right\}$. But this is just the condition which ensures that every eigenvalue $O \vec{i} \mathbf{C}$ has a negative real part (see, for example, BeIman [3]). Thus the same conäition which guarantees uniqueness also implies that $C$ is a stability matrix.

A case which might be consiajered as a generalization of the wo-person zero-sum game is the n-person "skew-symmetric" game where $c_{j i}=-C_{i j}^{\prime}, i, j=1, \ldots n$. For such a game we will have $\left[C+C^{\prime}\right]$ negative cefinite if $\left[C_{i i}+C_{i i}^{\prime}\right]$ is negative definite for $i=1, \ldots n$.

\section{3}




\section{Ciobal Stability of Equilibrium Point.}

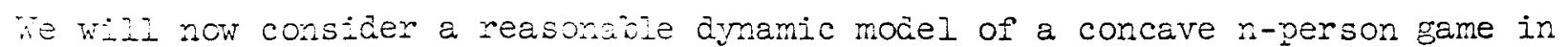
Wich each player changes his ow strategy in such a way that the joint strategy remains in $R$ and his own payoff function would increase if all other players hela to their current strategy. That is, each player changes his strategy at a rate proportional to the gradient with respect to his strategy of his payoff function, subject to the constraints. If we let the proportionality constant for the $i$ th player be $r_{i}$, we obtain the following system of difierential equiations for the strategies $x_{\dot{i}}$,

$$
\frac{\bar{a} x_{i}}{\bar{a}^{\dot{t}}}=\dot{x}_{i}=r_{i} \nabla_{i} \dot{0}_{i}(x)+\sum_{j=1}^{k} u_{j} \nabla_{i} h_{j}(x), \quad i=1, \ldots n
$$

where the vecior $u$ lies in a bounaed suisset $U(x)$ of the positive orthant of $E^{k}$. The effect of the summation term, with the appropriate choice of $u$, is to exsure that starting with any $x \in R$, the solution to (4.1) remains ir 2 . In fact, the right hand side of (4.1) is just the projection of the gradient $\nabla_{i} \rho_{i}(x)$ on the manifold formed by the active constraints at $x$. If we define an $m \times k$ matrix $\mathrm{H}(\mathrm{x})$, whose $j$ th column is $\nabla h_{j}(x)$,

$$
H(x)=\left[\nabla h_{1}(x) \quad \nabla h_{2}(x)--\nabla h_{k}(x)\right]
$$

and use the definition (3.9) of $g(x, r)$, we can define the mapping $f(x, u, r)$ of $i^{m+k} \rightarrow E^{m}$ for each fixed $\bar{r}>0$, as follows

$$
f(x, u, \bar{x})=g(x, \bar{r})+H(x) u
$$

Then the system (4.1) can be written

$$
\dot{x}=f(x, u, \bar{r}), \quad u \in v(x)
$$


Tine set $\quad \forall(x) \subset \Xi^{k}$ is aeterminea as pollows

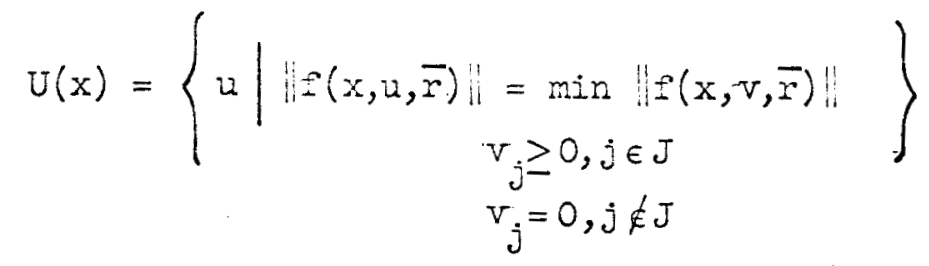

where

$$
J=J(x)=\left\{j \mid h_{j}(x) \leq 0\right\}
$$

Note that for every interior point $x$ of $R$ the set $J(x)$ is empty and $F(x)=0$, so thet $\vec{r}(x, i, \vec{r})=g(x, \bar{r})$.

We will assume that $g(x, r)$ and $H(x)$ are continuous in $x$ for all $x \in \overline{\hat{R}}$, where $\vec{R} \supset R$ is a compact set such that every point of the compact set $R$ is interior to $\bar{R}$.

Theorem 7

Starting at any point $x \in R$ a continuous solution $x(t)$ to $(4.4)$ exists, suig that $x(t)$ remains in $R \cdot$ for all $t>0$. a ProOI:

Because of the continuity in $x$, anc assuming only that $u$ is measurable in $t$, we have from the Carathéodory existence theory [14,16] that a continuous solution $x(t)$ exists for $x(t)$ in $\vec{R}$, which satisfies (4.4) almost everywere Now suppose that for some point $x^{\prime} \in \bar{R}$ on the trajectory $x(t)$ we have $h_{l}\left(x^{\prime}\right)<0$. Then by the continuity of $x(t)$ there must be an earlier point $\bar{x}$ on the trajectory, such that $h_{2}(\bar{x})=0$ an $\dot{h}_{2}(\bar{x})<0$. But from the latter $\operatorname{anc}(4.4)$ we have

$$
\dot{h}_{\ell}(\bar{x})=\nabla c_{l}^{\prime}(\bar{x}): \dot{x}=\nabla h_{l}^{\prime}(\bar{x}) \quad f<0
$$


ive iet the corresponding value of $u$ be $\bar{u} \in U(\bar{x})$. From the definition (4.3) tre have

$$
\|f\|^{2}=g^{\prime} g+2 \bar{u}^{\prime} H^{\prime} g+\bar{u}^{\prime} H^{\prime} H \bar{u}
$$

or

$$
\frac{\partial\|f\|^{2}}{\partial u_{\ell}}=2 \quad h_{\ell}^{\prime}(\bar{x})[g+H \bar{u}]=2 \nabla h_{\ell}^{\prime}(\bar{x}) f<0
$$

Accoraing to (4.9) we coula decrease the norm $\|$ f $\|$ by increasing $\bar{u}_{2}>0$. But since $b_{2}(\bar{x})=0$, we have $2 \in J(\bar{x})$ by $(4.6)$ ana therefore $\bar{u}$ carnot satisfy

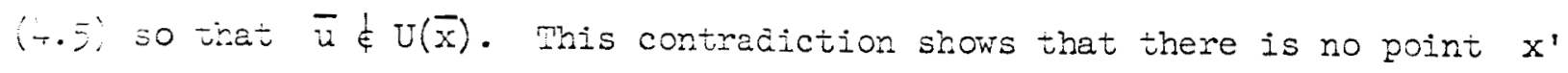
on the trajectory such that $h_{i}\left(x^{1}\right)<0$, for any $i$, which proves the theorem. By a airect application of the necessity of the Kuhn-Tucker concitions for the constrained minimization problem in (4.5) it is not difficult to demonstrate the following

Lemen

Ge ronzero elements of every vector $u \in U(x)$ are given by a vector $\vec{u} \in \Xi^{\vec{k}}, \bar{k} \leq k$, where

$$
\overline{\mathrm{u}}=-\left(\overline{\mathrm{r}}^{\prime} \overline{\mathrm{H}}\right)^{-1} \overline{\mathrm{H}}^{\prime} g(x, \overline{\mathrm{r}}) \geq 0
$$

The $\mathrm{m} \times \overline{\mathrm{K}}$ matrix $\overline{\mathrm{H}}=\overline{\mathrm{H}}(\mathrm{x})$ consists of $\overline{\mathrm{K}}$ linearly independent columns of $\mathrm{F}(\mathrm{x})$ selecteà from $\nabla \mathrm{h}_{j}(\mathrm{x})$ for $j \in J$. We now consider an equilibrium point $\bar{x}$ of the system of differential equitions (4.4). That is, for a fixed $r=\bar{r}$, we will call $\bar{x}$ an equilibrium point of $(4.4)$ if

$$
f(\bar{x}, u, \bar{x})=0, \quad u \in U(\bar{x})
$$


Fre system (4.4) will be callec asymptoticaliy stable in $R$ if for every initial point $x \in R$, the solution $x(t)$ to (4.4) converges to an equilibrium point $\bar{x} \in R$ as $t \rightarrow \infty$.

Theorem 8

If $R$ is given by (3.1) and $\left[G+G^{\prime}\right]$ is negative definite for $x \in R$, Where $G$ is the Jacobian of $g(x, \bar{r})$, then the system $(2.4)$ is as a stabie in $R$.

Proof:

The proof consists of showing that for $x$ and $u$ satisfying (4.4), the rave of change of $\|f(x, u, \bar{r})\|^{2}$ is always negative for $f(x, u, \bar{r}) \neq 0$. We first consiaer the situation when the selection of columns in $\vec{H}(x)$ remains unchangea. Then since all elements of $u$ are zero except those given by $\bar{u} \geq 0$, we have from (4.3)

$\operatorname{anc}$

$$
\hat{i}=g+\bar{E} \bar{u}=g+\sum \bar{u}_{j} \nabla h_{j}
$$

$$
\dot{f}=G \dot{x}+\sum \bar{u}_{j} Q_{j} \dot{x}+\bar{H} \dot{u}
$$

where $Q_{j}$ is the Jacobian of $\nabla h_{j}(x)$ (or its equivalent, the Hessian of $h_{j}(x)$ ) and is therefore negative semidefinite irom the concavity of $h_{j}(x)$. Now using (4.i3) ana $(4.4)$ we have

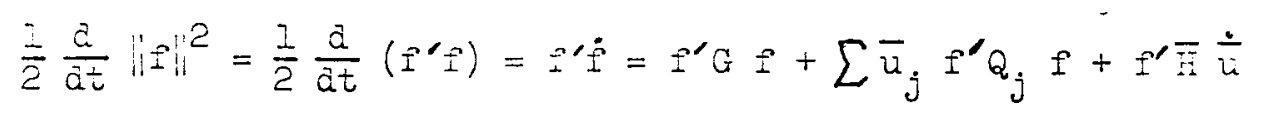

We consider the last term ana make use of (4.12) and (4.10) to show that

$$
f^{\prime} \dot{\bar{H}} \dot{\bar{u}}=\left[g^{\prime} \overline{\mathrm{H}}+\overline{u^{\prime}} \overline{\mathrm{H}}^{\prime} \overline{\mathrm{H}}\right] \dot{\overline{\mathrm{u}}}=\left[\mathrm{g}^{\prime} \overline{\mathrm{H}}-\mathrm{g}^{\prime} \overline{\mathrm{H}}\right] \dot{\overline{\mathrm{u}}}=0
$$




$$
\frac{l}{2} \frac{a}{d t}\|f\|^{2}=\frac{l}{2} f^{\prime}\left[G+G^{\prime}\right] \hat{I}+\sum \bar{u}_{j} f^{\prime} Q_{j} f \leq-\epsilon\|f\|^{2}
$$

for some $\epsilon>0$.

A change in the columns selecteen for $\bar{H}(x)$ can never increase the value of $\|$ "I. since the selection as determinea by $(4.5)$ will always minimize $\|f\|$. It therefore foliows from $(4.26)$ that $\lim \|=0$, so that $x(t) \rightarrow \bar{Y}$, where $\bar{x}$ $\pm \rightarrow \infty$

Is an equitionium point which sames $(4.11)$. By Theorem 7 , we have that $\bar{x} \in \bar{x}$, so tiat $(4.4)$ is asymptojicaily stable in $R$.

An ecuilibrium point $x^{0} \in R$ will be cailed globally asympto, ically stable in $R$ if for every starting point $x \in R$ the solution $x(t)$ to (4.4) converges to $x^{\circ}$. We will now show that with the appropriate concavity conditions the unique equilibrium point $x^{0}$ of $(2.1)$ is also globally asymptotically stable in र.

gareoris 9

IEt $R$ je Eiven by $(3.1)$ ara $G$ be the Jacobian of $g(x, r)$ for some ixxec $r=\bar{r}>0$. Then if $\left[G+G^{\prime}\right]$ is negative definite ior $x \in F$, the rormaizea equilibrium point, $x^{\circ}(\bar{r})$ is globally asymptotically stable in $R$. Proof:

Since $\left[G+G^{\prime}\right]$ is negative definite, $\sigma(x, \bar{x})$ is diagonally strictiy concave by Theorem 6 . Then by Theorem 4 there is a unique normalized equilibrium point $x^{0}=x^{0}(\bar{r})$, which satisfies (3.21), (3.22) and (3.23). But an ecuilibrium point $\bar{x}$ of (4.4) also satisfies these three relations. The first relation is satisied since $\bar{x} \in R$, while (4.11) is equivalent to (3.22) and (3.23). Finerefore we must have $\bar{x}=x^{0}$. By theorem 8 , the system $(4.4)$ is asymptenically 
stajie in R. Since $\bar{x}=x^{\circ}$ is urique the solution to (4.4) will converge to $x^{\circ}$ Prom every starting point in $R$, and the system is globally asymptotically stabie. 
5. Determination of Equilibrium Point.

The gicbal stability of the equilibrium point permits us to abtermine the unique equilibrium point for any concave game by appropriate mathematical programing computational methods. In particular, gradient methods for the concave nonlinear programming problem $[17,18]$ can be modified to find the equiliorium point for a concave game. Such methods take finite steps in the airection of the gradient of the function to be maximized taking account of the constraints by projection, or appropriate penalties, in order to remain in the feastbie resion $R$. The essential idea in applying one of these graiient methous to the concave game problem is to use the vector $g(x, r)$, given by (3.9), as if it were the gradient of a function of $x$, where the function is to be maximizea for $x \in R$. The solution to this "maximization" problem will give a point $x^{\circ} \in R$ where the Kuhn-Tucker conaitions (3.21), (3.22) and (3.23) are satisfiea. But as has been shown such a point is the unique equilibrium point for the concave game. Note that the optimality conditions involve only ine gradient $g(x, r)$ and do not recuire that the function itself be znown. Tre gacient projection method car be considerea as a finite diffenence Exproximation to the system (4.4), where the solution is obtainea by a sequence of finite steps in the direction of the projected gradient $f(x, \vec{u}, \vec{r})$. The only practical difference between this and a true maximization probiem is that in the latter case we choose the step length so as to give a maximum of the trie function value along the chosen ray, whereas for the equilibrium point problem we choose the step length so as to minimize the norm of $f$.

To show how this is done we consider the finite difference approximation to $(4.4)$ given by

$$
x^{j+1}=x^{j}+\tau^{j} f\left(x^{j}, u^{j}, \bar{r}\right), \quad u^{j} \in U\left(x^{j}\right)
$$

where $\tau^{j}$ is the step length to be selected. 


\section{peorem 20.}

If the assumptions of Theorem 8 are satisfied then a finite step length

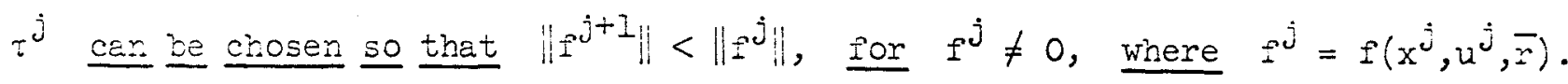
Proof:

For $u=u^{j}$ held fixed we have

$$
\overline{\mathbf{I}}^{j+1}=f\left(x^{j+1}, u^{j}, \bar{r}\right)=f^{j}+\bar{F}\left(x^{j+1}-x^{j}\right)
$$

witere $\bar{F}$ is a mean value of the Jacobian of $f$, so that $\hat{f} \bar{F}=0$, For $f \neq 0$. Then from (5.1) we have

$$
\overline{\mathfrak{I}}^{j+I}=\left(I+\tau^{j} \bar{F}\right) f^{j}
$$

The norm of $\overline{\hat{I}}^{j+1}$ is minimized by the choice

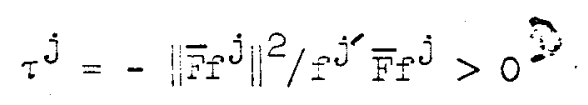

which gives

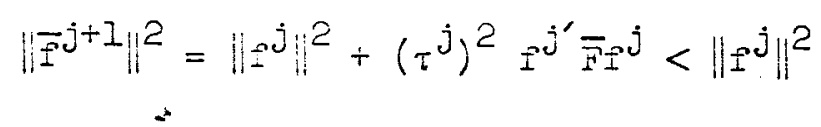

Finally since $f^{j+1}=f\left(x^{j+1}, u^{j+1}, \bar{r}\right)$, where $u^{j+1} \in U\left(x^{j+1}\right)$, it follows from (4.5) ana (5.2) that $\left\|f^{j+I}\right\| \leq\left\|\hat{i}^{j+I}\right\|<\left\|f^{j}\right\|$.

The convergence of this firite difierence procedure to the urique equilibrium point $x^{\circ}$ can be show as in theorem 8 . 


\section{References}

3. Amot, K. J. and G. Debreu, "Tixistence of an equilibrium for a competitive economy", Econometrica 22, (1954), pp. 265-290.

2. L. Hurwicz and J. Juawa, "Constraint qualifications in maximization problems", Nav. Res. Log. Qu. E, (1959), pp. 175-191.

3. Eellman, R., Introauction to Matrix Araijsis, McGraw Hill, (1960), p. $242-243$.

4. Kakutari, S., "A generalization of Brouwer's fixea point theorem", Duke Vish. j. 8, (1941), pp. 457-458.

5. Kariin, S., Veihematical Vethoas ana Theory in Games, Prograrming and Econcrics, Vol. II, Aâaison-Vesiey, (i959), pp. 14-15.

6. Kunn, on Vetrematical Statistics ana Probability, Univ. of Calif. Press, (1951), pp. $481-492$.

7. "An algorithm $\vec{i}$ or equilibrium points in bimatrix games", Proc. Nat. Acad. Sci. U.S.A. 47, (1961), pp. 1657-1662.

3. Yangasarian, O. I. and H. Stone, "Two-person nonzero-sum games and quaâratic zosramming", To appear J. Vzun. Anal. and Applict, 1964.

9. Yckenzie, I. W., "On the existence or general equilibrium for a competitive manket", Econometrica 27, (1959), pp. 54-71.

10. Nash, J.F., "Equilibrium poinis in n-person games", Proc. Nui. Acaä. Soi. U.S.A. 36, (1950), pp. $48-49$.

11. W. "Non-cooperative Ǧmes", Annals of Vath. 54, (1951), pp. 286295.

12. Shapley, I. S., "A solution containing an arbitrary closed component", Annals of Math. Stuaies No. Lo, Princeton Univ. Press, (1959), pp. 87-93.

13. Arrow, K. J., L. Hurwicz and $\mathrm{H}$. D. Blcek, "On the stability of the competitive ecuilibrium, Parts I anō II", Eonometrica 26, (1958), pp. 522-5!2 and 27 (1959), pp. 82-109. 
… Loäirston, E. A. and N. Levinson, Theory of Oräinary Differential Equations, Nocraw Hill (1955), Chap. 2.

5. Cale, D., The Theory of Linear Economic Models, McGraw Hill (1960) Chap. 7.

Z6. Pontryagir, L. S., V. G. Boltyanskii, R. V. Gamkrelidze and I. F. Mischenko, Ne Vethematical Theory of Optimal Processes, Interscience (1962), Section 10.

2?. Foser, J. B., "The gradient projection method for nonlinear programming, Parts I anà II", J. Soc. Incuust. Appl. Math. $\underline{8}$ (1960) pp. 181-217 anä $\underline{9}$ (196́i), pp. 5i4-532.

16. Facco, A. V. and G. P. NcCormick, "Prusaming under nonlinear constraints by unconstrainec inimization. A primal-äual methoa", RAC-TP-96, The Eesearch Analysis Corp., Sept. 1963. To appear Vanagement Science, Jan. 1964.

29. Uzawa, E., "Mhe stability of dynamic processes", Econometrica 29, (196I), 2p. $617-630$. 